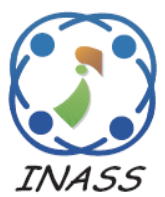

\title{
Haze Removal of a Single Image by Using the Brightness Prior
}

\author{
Monika Verma $^{1 *}$ Vandana Dixit Kaushik $^{1} \quad$ Vinay Pathak $^{1}$ \\ ${ }^{I}$ Computer Science and Engineering Department, Harcourt Butler Technical University, Kanpur, India \\ * Corresponding author's Email: mvmonikaverma261@gmail.com
}

\begin{abstract}
In this paper a novel method for removing haze is given which is based on the Hue Saturation Value color model together with dark channel subtraction. Single image fog/haze removal is a challenging task due to its ill posed nature. As fog/haze increases the brightness of the images captured also increases. In the current wok, a new methodology has been used which involves the subtraction of extra image brightness from the hazy images for keeping the hue and saturation intact. It uses the information of image brightness to get the clear image by histogram equalization. Together, the three approaches of HSV color model, histogram equalization and Dark Channel Subtraction gives clear images. Experimental results are shown in comparison with the state of the art methods. The approach used in the current paper is at par with the current methodologies and the results are better than the existing approaches.
\end{abstract}

Keywords: Atmospheric scattering model, Hue saturation value color model, Histogram equalization, Dark channel subtraction.

\section{Introduction}

The outdoor images taken in a bad weather suffer from various degradations caused due to loss of contrast and also due to more brightness of pixels. Fog/haze is a phenomenon where in the electromagnetic light is scattered due to suspended particles in the atmosphere. The result of which is that the viewer is not able to fully comprehend the actual features of the scene.

The presence of haze in the atmosphere degrades the quality of images captured by visible camera sensors. The removal of haze, called dehazing, is typically performed under the physical degradation model, which necessitates a solution of an ill-posed inverse problem. [1] The task of image dehazing is often time consuming. It leads difficulty in the analysis of segmenting the foreground and the background images in image processing. The poor visibility influence the accuracy of the vision systems. The images captured are not clear due to which many of the tasks of either the automatic monitoring or remote sensing analysis are not up to the mark. Even the image classification tasks and the recognition tasks face difficulties.

The basic idea of removal of fog/haze is to solve the ambiguity in the equation caused due to more number of unknown variables than the known ones used to get a fog/haze free image. Research in defoggging of images or videos has been growing up with the upcoming of more efficient algorithms. In the early researches the defogging used the conventional approach of histogram processing to remove the haze $[2,3]$. The single image was not sufficient to provide the information needed to remove haze completely. Later the improvement came when multiple images tend to solve the ambiguity by acquiring more number of known variables. The same image was being viewed under different settings. In polarization based methods two images of the same scene are taken into consideration $[4,5]$.The images taken are with different degrees of polarization. Getting two strictly aligned images is problematic situation. Also they tend to increase the noise and are not beneficial for visibility [6]. The other methods that uses multiple images is based on varying atmospheric conditions. 
Two images of the same scene are considered with the same depth and same reflectance. Here also getting two images that are strictly aligned is a major problem [7, 8]. In [9], the authors proposed a parallel image haze remove algorithm, adopting optimized contrast enhancement approach, to optimize the performance based on GPU platform. The approach followed in [10] uses the given depth information to remove haze. In [11] the authors have compared the state-of-the-art in this area and puts forwards their strengths and weaknesses. Through experiments the efficiencies and shortcomings of these algorithms are shared. Using the single image to solve the ambiguity of the equation of the defogging model involves the knowing of some priors. These priors can be anything ranging from statistical properties to some application based rules. The assumptions are made in order to get more number of known variables to solve the fog/haze equation. Tan considered the prior based on the local contrast of the image [12]. By using the Markov Random Field the method maximizes the local contrast of the image. The visibility is enhanced but the image looks oversaturated.

In the dark object subtraction method the assumption is that there must be a dark object in at least one of the three channels. This method is applicable in satellite images where the depth of the scene points can be taken as constant [13].A statistical method is used by Fattal wherein the approach of ICA tends to remove haze from the colour images [14].It has difficulties in removing haze from gray scale image and also the dense haze images cannot be dealt with such an approach. A simple and effective technique of Dark Channel Prior has been proposed by $\mathrm{He}$ et al. in which the thickness of the haze is estimated and as a result of which the haze free results are restored. With the computationally intensive method the sky images are not well handled [15]. In [16] the authors have proposed a novel and effective algorithm for single image fog removal that's capable of handling images of gray and color channels. The proposed algorithm introduces Dark Channel Prior (DCP) followed by Weighted Least Square (WLS) and High Dynamic Range (HDR) based fog removal scheme. Nishino et al used Markov Random Field to approximate the scene radiance [17].Meng et al. proposed the regularization dehazing approach to get back the original image [18]. Tang et al. combined the four types of haze features with Random Forest Transmission [19]. The authors in [20] have used the single image color attenuation prior. Inspite of the fact that these [16-20] methods are showing the remarkable progress, the limitation of the state-of-the-art methods lies in the fact that the haze-relevant priors or heuristic cues used are not effective or efficient enough.

In this paper the beforehand knowledge used is the fact that the brightness of the pixels in a hazy environment increases in proportion with the degree of fog/haze. The simple and the powerful prior helps to create a model in which all the three channels of hue, saturation and brightness are found out. The effectiveness of this method is considerably high. The dehazing method is superior to the existing menthods and the images obtained are of good quality. More is the fog/haze more is the brightness of the pixels in the image. The difference between the hue and the brightness increases with the proportion of fog/haze.

The paper is divided into the following sections. Section 2 describes the fundamental Atmospheric Scattering Model. Section 3 discusses the novel approach. The image reconstruction is done by removing the fog from the images. Enhancement is done by using the concepts of HSV color model. Section 4 provides the experimental results. Image quality is evaluated by some standard metrics. In section 5 the conclusions and the future scope are summarized.

\section{Methodology}

The Atmospheric Scattering Model proposed by McCartney is used to find out the fog/haze free image[21]. The fog/haze equation is given by

$$
\begin{aligned}
& \boldsymbol{I}(\boldsymbol{x})=\boldsymbol{J}(\boldsymbol{x}) t(\boldsymbol{x})+\boldsymbol{A}(1-t(\boldsymbol{x})) \\
& t(\boldsymbol{x})=e^{-\beta d(\boldsymbol{x})}
\end{aligned}
$$

where $\boldsymbol{x}=(x, y)$ is a $2 \mathrm{D}$ vector, $\boldsymbol{I}(\boldsymbol{x})$ is a $3 \mathrm{D}$ RGB vector of the color at a pixel(pixel of the hazy image), $\boldsymbol{J}(\boldsymbol{x})$ is a $3 \mathrm{D}$ RGB vector of the color of the light reflected by the scene point at $\boldsymbol{x}$ (pixel of a fog/haze free image). $\mathbf{J}$ is known as the scene radiance, $t$ is a map called transmission or transparency of the fog/haze. $t(x)$ is a scalar in $[0,1]$, $\beta$ represents the scattering coefficient of the atmosphere, $d(x)$ is the depth of a particular pixel. The term $\boldsymbol{J}(\boldsymbol{x}) t(x)$ is known as direct attenuation. Attenuation is due to the particles in the atmosphere. The particles are providing hindrance in the way of light. They don't allow the entire light to fall on the object as a result of which the light that is reflected from the objects is lower than what they would have reflected when the scene was fog/haze free. Because of direct attenuation the brightness of the scene objects decreases in a multiplicative manner. The transmission term is the ratio of light that is reaching 
the observer without getting attenuated to the light that is reflected by the object.

The term $\boldsymbol{A}(1-t(x))$ is known as the airlight[22]. Due to the Airlight the brightness of the scene increases because in the first place the particles are absorbing the light and then they tend to scatter light in different directions thus becoming the tiny sources of light that keep floating in the atmosphere. The airlight increases in proportion to the amount of fog/haze. So overall the effect of airlight is more than direct attenuation because of which the brightness of the hazy image is much more and on the other hand the hue and the saturation are having low values.

To solve the above equation the most important information is the depth information of the pixels. Once the depth is found out the transmission becomes an easy parameter to fetch as the term $\beta$ which is known as the scattering coefficient is assumed to be constant in homogeneous atmospheric conditions. To find out the parameter of airlight we notice that once the transmission is zero then the equation is reduced to Eq. (3).

$$
I(x)=A
$$

As shown in Eq. (4), the transmission parameter becomes zero if the depth of a pixel $(d(x))$ is infinite.

$$
t(x)=e^{-\infty}=1 / e^{-\infty}=0
$$

Geometrically the fog/haze equation describes the transmission $t$ as the ratio of the two line segments

$$
t(x)=\frac{\|\boldsymbol{A}-\boldsymbol{I}(\boldsymbol{x})\|}{\|\boldsymbol{A}-\boldsymbol{J}(\boldsymbol{x})\|}
$$

In RGB color model the vectors $\boldsymbol{A}, \boldsymbol{I}(\boldsymbol{x})$ and $\boldsymbol{J}(\boldsymbol{x})$ are coplanar and their corresponding end points are collinear.

\section{Novel approach to get clear images}

\subsection{Color model (HSV)}

The color model emphasizes the specification of colours and the subspace within the coordinate system where each color is represented by a single point. The way the human perceives the color object is described by hue, saturation and brightness. Hue refers to the color to which it resembles. E.g. all shades of red have the same hue. Hues are described by a number that specifies the position of the corresponding pure color on the color wheel, as a fraction between 0 and 1 .
The saturation of a color describes of how white the color is. A pure blue is fully saturated and white has a saturation value of zero. The value of a color describes how dark a color is. A value of white is one with increase in the darkness as the colours keep moving away from the white. A single hexcone color model is shown in figure given below.

The wheels outer edge of the cone is known as the color wheel. It indicates all the pure colours. The parameter hue indicates the angle around the wheel. The saturation is zero for any color on the axis of the cone. The centre of the top circle is white. An increase in the value of $\mathrm{S}$ corresponds to a movement away from the axis. The value is one for white. A decrease in the value of $\mathrm{V}$ corresponds to a movement away from white and toward the top of the cone [23].

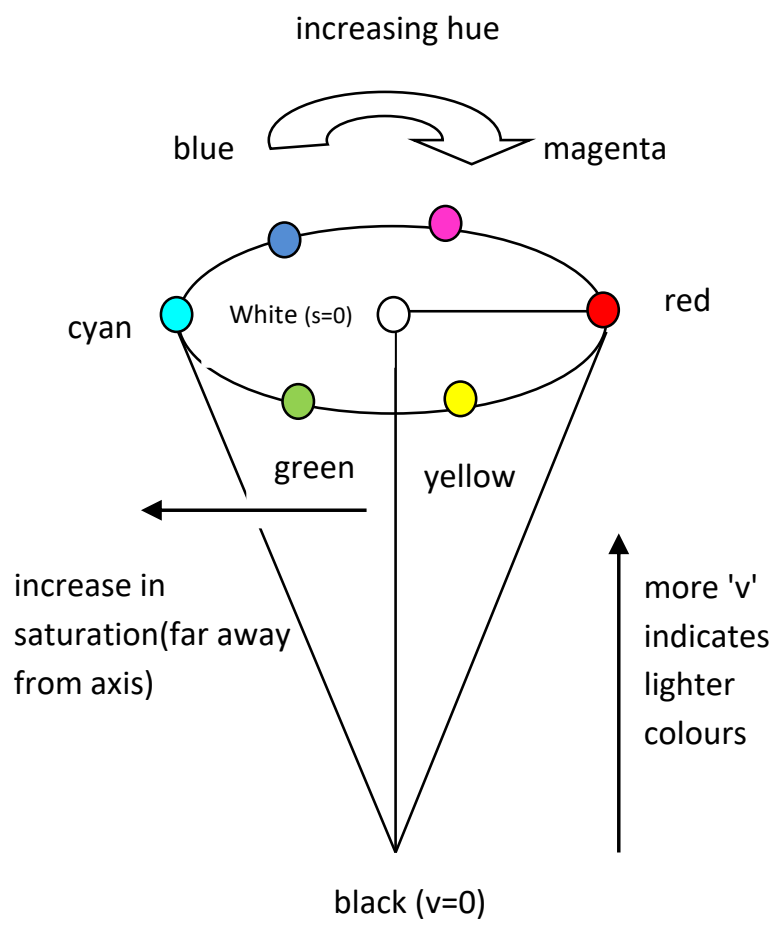

Figure.1 The hexcone color model

\subsection{Histogram equalization}

Histogram equalization is a technique to enhance the contrast of an image by adjusting the image intensities. Let $\mathrm{g}$ be an image with the pixel intensities lying in the range of 0 to $\mathrm{L}-1$ where $\mathrm{L}$ is the gray values of the image. Let $\mathrm{q}$ denote the normalised histogram of $\mathrm{g}$. Therefore

$$
q_{n}=T_{n} / T_{p}
$$

where $T_{n}$ is the no of pixels with gray values of $n$ and $T_{p}$ is the total number of pixels where value of $n$ 
lie in the range of 0 to L-1. The histogram equalized image is defined by

$$
\text { hist }_{e q}=\text { floor }\left((L-1) \sum_{n=0}^{g_{(i, j)}} q_{n}\right)
$$

\subsection{Dark channel subtraction (DCS)}

In a hazy image the probability that some pixels have a very low intensity (shadows, dark objects, colourful objects )in one of the color channel. The intensity component in the dark channel is an indication of the airlight. So the dark pixels can provide estimation of haze/fog transmission. If $\mathbf{J}$ is an image then the dark channel $\left(\mathrm{D}_{\mathrm{j}}\right)$ is defined as

$$
D_{j}=\min _{(b \epsilon r, g, b)}\left(\min _{y \in \Omega(x)}\right)\left(J^{b}(y)\right)
$$

where $\mathrm{J}^{\mathrm{b}}$ is the color band of $\mathrm{J}$ and $\Omega(x)$ and is the patch whose centre is at $\mathrm{x}$. Due to the component of airlight $\mathbf{A}(1-\mathrm{t}(\mathbf{x}))$ a foggy/hazy image has more brightness than its fog/haze free version. The intensity calculated of the dark channel determines the thickness of the fog/haze. By using the Dark channel subtraction method, the work presented in this paper has found the components of hue and saturation, after the conversion of RGB to the HSV model. To find out the transmission the assumption is made that it is constant in a particular patch. The transmission is given by

$$
t(x)=1-\min _{b}(\min )_{y \in \mathcal{L}(x)}\left(I^{b}(y) / A^{b}\right)
$$

To estimate the atmospheric light(A) the brightest of the pixels in the dark band is considered. ' $b$ ' is the color band. The value of the brightest pixels is the airlight. If the value of the transmission $t(x)$ is close to zero the term $J(\boldsymbol{x}) t(\boldsymbol{x})$ which is direct attenuation, also tends towards zero. The value of scene radiance is prone towards noise. Therefore $t(\boldsymbol{x})$ is restricted to the lower bound $t_{c}$. The value given to $t_{c}$ is .1.The scene radiance is recovered by the equation

$$
\boldsymbol{J}(x)=\frac{\boldsymbol{I}(\boldsymbol{x})-\boldsymbol{A}}{\max \left(t(\boldsymbol{x}), t_{c}\right)}+\boldsymbol{A}
$$

"Value" in HSV model plays an important part in the hazy or the foggy weather. As the fog/haze increases correspondingly the brightness component also increases. It would be of great help if the brightness component is spread out equally in the image. The hue and the saturation is then modelled by the dark object subtraction. So together we have modelled the hue, saturation and the value components in two phases. In the first phase the coloured image is transformed into the HSV model and then after doing the histogram equalization on the value component the new and transformed value of the brightness is found. In the next phase the new and the transformed values of hue and saturation are found out. By applying the dark channel subtraction the hue and the saturation values are found out.

Fig. 2 given below represents the proposed approach. Out of the three columns the first column is representing the first phase from which the 'value component' is extracted. The Input foggy image is taken. It is converted to HSV space from the RGB space. Histogram equalization is done after the extraction of the brightness component of the image. The second column represents the second phase from which the hue and saturation values are extracted after applying the dark channel subtraction method. The third column represents the combination of the two approaches and the final result which is a fog/haze free image.

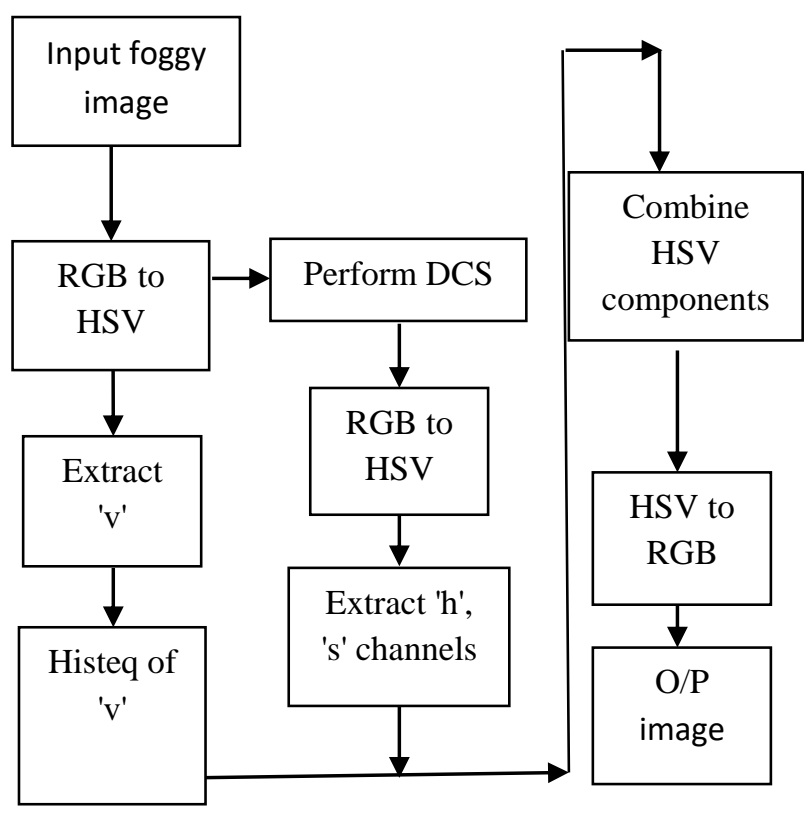

Figure.2 The diagrammatic representation of the proposed work

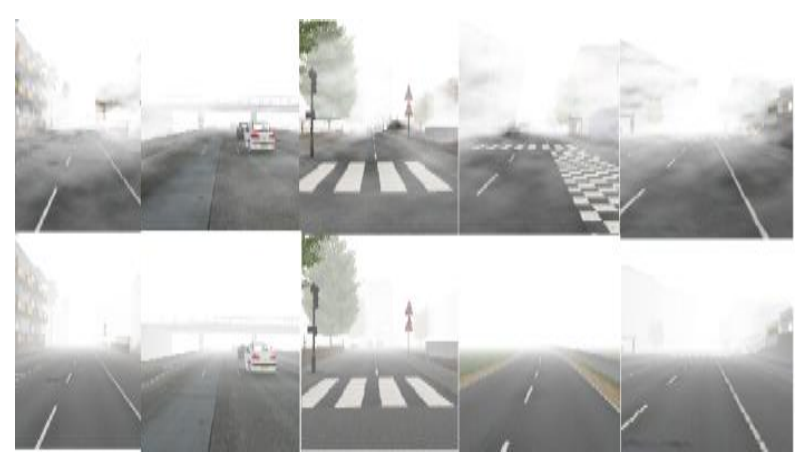

Figure. 3 The synthetic hazy images, first row is representing the heterogeneous hazy images, the second row is representing homogeneous hazy images. 


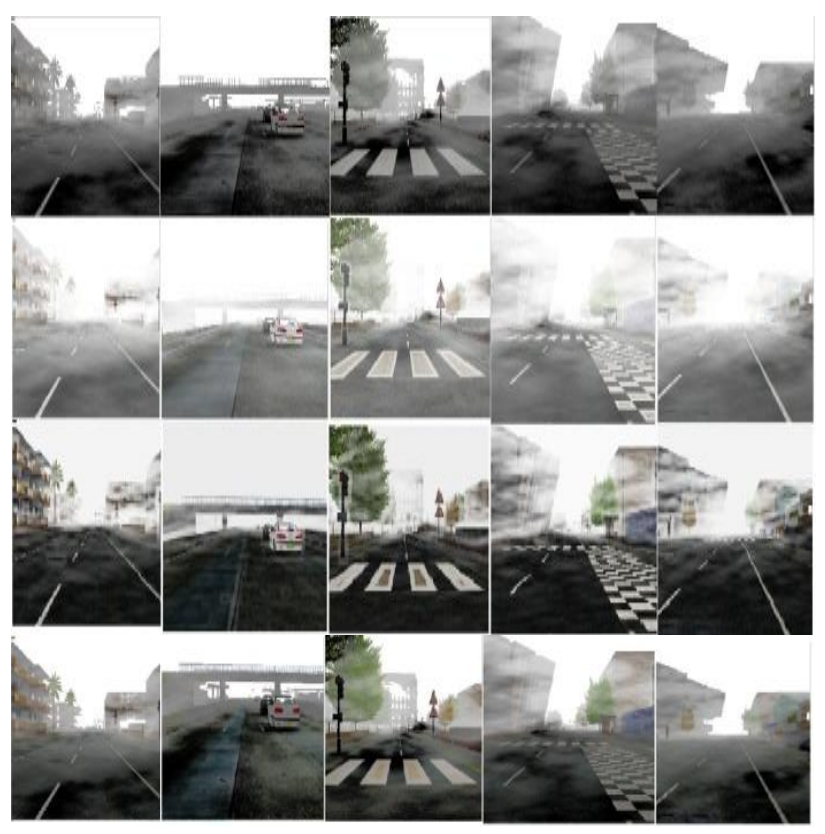

Figure.4 Images of heterogeneous hazy images after applying the algorithms, first row is the proposed algorithms result of first phase, second row is the results of algorithm of Quinsong et al., third row is the result of algorithm of He et al., fourth row is the proposed algorithm result of the final phase.

Given above are the images of fog/haze. Row 1 of Fig. 3 corresponds to the heterogeneous images and row 2 corresponds to the homogeneous images. The data set used is FRIDA Data Set [24, 25]. It consists of synthetic hazy images. This paper has considered only two types of hazy images homogeneous and heterogeneous.

Fig. 4 corresponds to the results generated from the proposed work (first phase), the algorithms of Zhu et al. and $\mathrm{He}$ et al. respectively. The first row which corresponds to the results of the proposed work after just applying histogram equalization on the "value component" of the HSV model of the input hazy images shows the great clarity in comparison to the results that are generated by the latter algorithms. Second row corresponds to the results of Zhu. As it can be seen that the fog/haze is too much visible in the second row as compared to the first one.

In the third row, the algorithm proposed by He et al. the blocking artefacts as well as the clarity of the image as the depth increases can be well seen which are not there in the first row, the proposed work. The images in the first row are better than the existing algorithms in terms of the Structural Similarity Index, Mean Square Error, Peak Signal to Noise Ratio, and the max error. The colours are not well seen in the proposed work, the image tends to be more towards the gray scale.

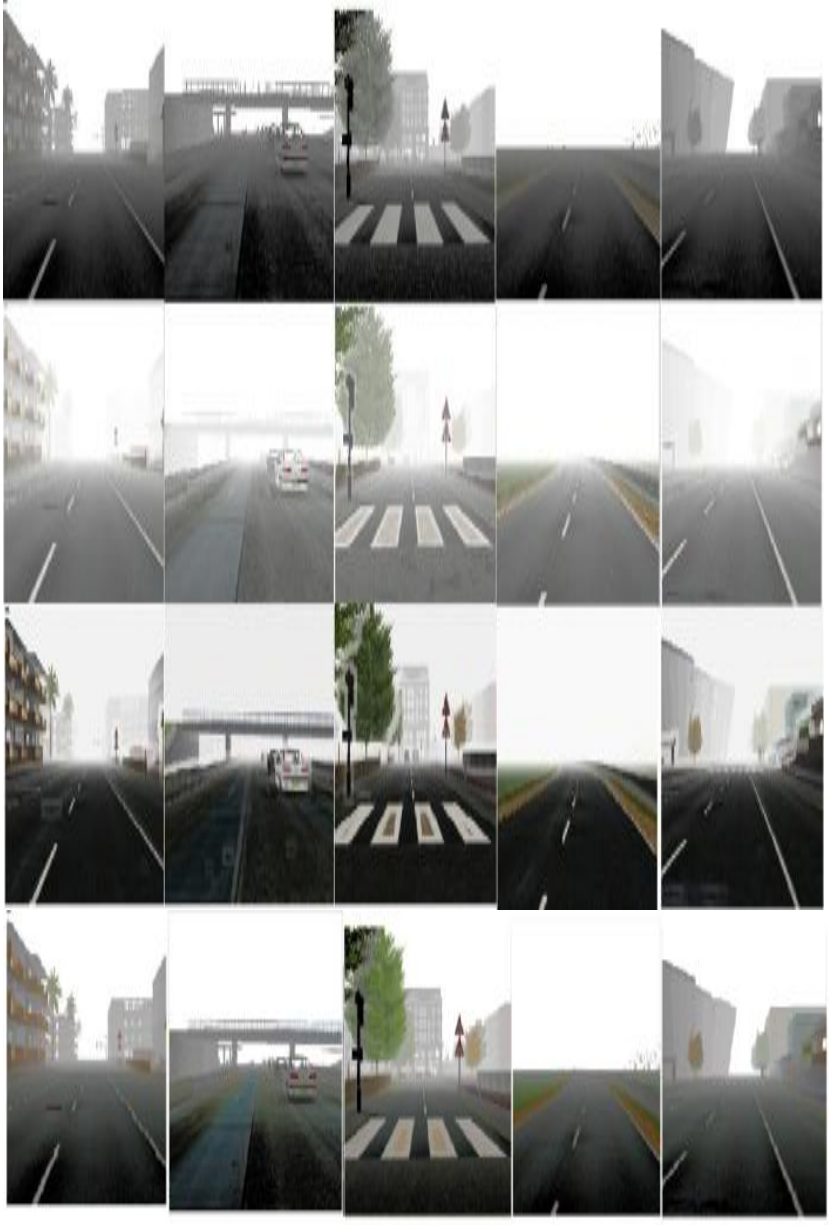

Figure.5 Images of homogeneous hazy images after applying the algorithms, first row is the proposed algorithms result of first phase, second row is the results of algorithm of Quinsong et al., third row is the result of algorithm of He et al., fourth row is the proposed algorithm result of the final phase.

To overcome this limitation the hue and the saturation channel can be well generated from the dark channel subtraction method. The resultant image can be seen in the fourth row in which the clarity as well as the colours and the saturation are appropriate.

The first row of Fig. 5 shows the output of the proposed algorithm (the first phase) on the five images of the second row of Fig. 3. The second and the third row denotes the results after applying the Zhu et al. and He et al. approach respectively. The fourth row denotes the final output of the algorithm applied on the foggy images.

In the proposed approach the blocking artefacts are not there as they can be seen in the algorithmic approaches followed by He et.al. [15] and Zhu et.al. [20]. In addition the far away objects from the camera are not well seen in [15] and [20].On the other hand, through the approach used in this paper, one is able to clearly see the clarity of the images 
even if the distance is large from camera and the objects are covered with haze.

\section{Experimental results}

The table given below compares the three given algorithms in terms of maxerr, mse and the PSNR values. The ten images taken are the same as given in Fig. 3. Out of the ten images first five images are heterogeneous images and the rest (6-10) are the homogeneous images. The input foggy images have a large error and a low Peak Signal to Noise Ratio. The approach suggested by Zhu et al and He et al. reduces the mean square error and increases the Peak Signal to Noise Ratio. The proposed work shows a slight betterment in the results, either it is the mean square error (MSE) or the Peak Signal to Noise Ratio (PSNR) or the Structural Similarity index of Images (SSIM).

The FRIDA data set $[24,25]$ is used that contains the foggy synthetic images. The high maxerr corresponds to the fact that the error as compared to the ground truth is at its maximum. A low value of maxerr indicates the image is moreclose towards the ground truth. The quantitative assessment is done by using these four parameters given below:

\subsection{Mean Square Error}

If comparison of two images is made based on their respective qualities then a metrics called Mean Square Error is used. The MSE between two images $\mathrm{g}(\mathrm{x}, \mathrm{y})$ and $\mathrm{f}(\mathrm{x}, \mathrm{y})$ is

$$
e_{M S E}=1 / M N \sum_{n=1}^{M} \sum_{m=1}^{N}[f(n, m)-g(n, m)]^{2}
$$

where $M$ and $N$ represent the number of rows and columns respectively.

\subsection{Peak signal to noise ratio}

The peak signal to noise ratio avoids one of the problems with the MSE (MSE depends strongly on the image intensity scaling) by scaling MSE according to the image range.

$$
P S N R=-10 \log _{10} \frac{e_{M S E}}{Q^{2}}
$$

where $Q$ is the maximum pixel value.

\subsection{Maxerr}

MAXERR is the maximum absolute squared deviation of the data from the approximation.

\subsection{Entropy}

Entropy a scalar value representing the entropy of grayscale image I. Entropy is a statistical measure of randomness that can be used to characterize the texture of the input image. Entropy is defined as

$$
-\sum_{i=1}^{i=n} p\left(x_{i}\right) \log _{2} p\left(x_{i}\right)
$$

where $p$ contains the histogram counts returned from imhist. By default, entropy uses two bins for logical arrays and 256 bins for uint8, uint16, or double arrays. I can be a multidimensional image. If I has more than two dimensions, the entropy function treats it as a multidimensional gray scale image and not as an RGB image.

Table 1 shows the comparison of results in terms of MAXERR and MSE of the images taken by various authors. The proposed work shows less values for MAXERR and MSE in comparison to the values for existing approaches.

Table 2 indicates the results in terms of PSNR and ENTROPY of the various images taken by the different authors. As indicated the values of the metrics are better for the proposed approach than for the existing techniques.

The proposed approach is better than the existing approaches as at the very first place histogram equalization is done. This spreads the fog almost equally in all directions. Then the extra image brightness is removed by finding the dark channel pixels. When the dark channel are found out then the channel is subtracted from the foggy image.The proposed approach works better than the methods specified by He et.al. [15] and Zhu et.al. [20]. In addition the far away objects from the camera are not well seen in $[15,20]$.On the other hand, through the approach used in this paper, one is able to clearly see the clarity of the images even if the distance is large from camera and the objects are covered with haze.

\section{Conclusion and future scope}

Single image haze removal is one of the important tasks in image processing through which the clarity of the images is greatly increased. The clarified images can be used in a number of applications which include traffic monitoring, real time target tracking, video surveillance, target recognition, fracture detection in medicine, satellite remote sensing, and driverless vehicle technology. In the present work a novel approach based on the Hue Saturation Value color model together with 
Table 1. Shows the comparison of MAXERR and MSE of the resultant images of different authors

\begin{tabular}{|c|c|c|c|c|c|}
\hline \multirow{3}{*}{ Image 1} & & Hazy & Zhu et el.[20] & He et al. [15] & Proposed \\
\hline & Maxerr & 253 & 251 & 233 & 233 \\
\hline & Mse & 7500 & 6800 & 3300 & 2600 \\
\hline \multirow{2}{*}{ Image 2} & Maxerr & 252 & 251 & 229 & 230 \\
\hline & Mse & 2730 & 2680 & 1600 & 1180 \\
\hline \multirow{2}{*}{ Image 3} & Maxerr & 241 & 241 & 226 & 231 \\
\hline & Mse & 8030 & 6900 & 4210 & 4000 \\
\hline \multirow{2}{*}{ Image 4} & Maxerr & 243 & 242 & 234 & 236 \\
\hline & Mse & 10200 & 9240 & 4750 & 4150 \\
\hline \multirow{2}{*}{ Image 5} & Maxerr & 248 & 248 & 236 & 231 \\
\hline & Mse & 7960 & 7270 & 3900 & 2800 \\
\hline \multirow{2}{*}{ Image 6} & Maxerr & 254 & 255 & 233 & 220 \\
\hline & Mse & 7690 & 7210 & 3730 & 2500 \\
\hline \multirow{2}{*}{ Image 7} & Maxerr & 252 & 251 & 226 & 242 \\
\hline & Mse & 3260 & 2980 & 1710 & 1390 \\
\hline \multirow{2}{*}{ Image 8} & Maxerr & 242 & 241 & 233 & 190 \\
\hline & Mse & 8780 & 7610 & 4340 & 3780 \\
\hline \multirow{2}{*}{ Image 9} & Maxerr & 230 & 224 & 224 & 224 \\
\hline & Mse & 11200 & 12100 & 12000 & 11000 \\
\hline \multirow{2}{*}{ Image 10} & Maxerr & 248 & 248 & 236 & 240 \\
\hline & Mse & 8450 & 7900 & 4290 & 2630 \\
\hline
\end{tabular}

Table 2. Shows the comparison of PSNR and ENTROPY of the results of different authors

\begin{tabular}{|c|c|c|c|c|c|}
\hline \multirow{3}{*}{ Image 1} & & Hazy & Zhu et el.[20] & He et al. [15] & Proposed \\
\cline { 2 - 6 } & PSNR & 9.36 & 9.76 & 12.95 & 13.96 \\
\cline { 2 - 6 } & Entropy & 6.0 & 6.0 & 6.3 & 5.3 \\
\hline \multirow{2}{*}{ Image 2 } & PSNR & 13.76 & 13.85 & 16.08 & 17.42 \\
\cline { 2 - 6 } & Entropy & 5.0 & 5.0 & 5.2 & 4.6 \\
\hline \multirow{2}{*}{ Image 3 } & PSNR & 9.08 & 9.72 & 11.89 & 12.10 \\
\cline { 2 - 6 } & Entropy & 6.3 & 6.3 & 6.7 & 5.9 \\
\hline \multirow{2}{*}{ Image 4 } & PSNR & 8.03 & 8.47 & 11.37 & 11.95 \\
\cline { 2 - 6 } & Entropy & 6.4 & 6.5 & 6.8 & 5.9 \\
\hline \multirow{2}{*}{ Image 5 } & PSNR & 9.12 & 9.52 & 12.79 & 13.66 \\
\cline { 2 - 6 } & Entropy & 5.9 & 6.0 & 6.0 & 5.3 \\
\hline \multirow{2}{*}{ Image 6 } & PSNR & 9.27 & 9.55 & 12.41 & 14.15 \\
\cline { 2 - 6 } & Entropy & 5.8 & 5.9 & 5.9 & 5.2 \\
\hline \multirow{2}{*}{ Image 7 } & PSNR & 13.0 & 13.39 & 15.80 & 16.70 \\
\cline { 2 - 6 } & Entropy & 4.9 & 4.9 & 5.2 & 4.5 \\
\hline \multirow{2}{*}{ Image 8} & PSNR & 8.70 & 12.36 & 11.75 & 12.36 \\
\cline { 2 - 6 } & Entropy & 6.1 & 6.1 & 6.3 & 5.8 \\
\hline \multirow{2}{*}{ Image 9 } & PSNR & 7.64 & 7.29 & 7.15 & 7.70 \\
\cline { 2 - 6 } & Entropy & 4.3 & 4.2 & 4.1 & 3.8 \\
\hline \multirow{2}{*}{ Image 10 } & PSNR & 8.86 & 9.16 & 11.81 & 13.93 \\
\cline { 2 - 6 } & Entropy & 5.7 & 5.9 & 5.9 & 5.1 \\
\hline
\end{tabular}

dark channel subtraction is used. The proposed approach works better than the existing approaches. The method involved converts the input foggy image from the RGB to the HSV space. The proposed algorithm takes the histogram equalized values of the ' $\mathrm{V}$ '(value) component. Once the value component is known the hue and the saturation components are found by the standard algorithm of dark channel subtraction. Together now the image has all the three components of hue, saturation and value. The HSV space is now converted to RGB space again, to get a clear image. The image got from the proposed approach has PSNR, maxerr, entropy, MSE values better than the algorithms proposed by He et al. [15] and Zhu et al.[20]. 
In solving the atmospheric model the term beta (scattering coefficient) is taken to be constant as the atmospheric conditions are taken to be homogeneous. Keeping the above scenario in mind the histogram equalization of the brightness component is done. Instead of histogram equalization if some algorithm is developed where the in heterogeneity of the atmosphere is taken into consideration as well as the scattering coefficient can be found out then it would have more flexibility than the existing approaches. Moreover the view tends to be more inclined towards higher values of the gray levels. These three drawbacks can be considered as the future scope of the work.

\section{References}

[1] S.Lee, S.Yun, J.H. Nam, C.S. Won, and S.W. Jung, "A review on dark channel prior based image dehazing algorithms", EURASIP Journal on Image and Video Processing, Vol. 1, No. 4, 2016.

[2] J.A. Stark, "Adaptive image contrast enhancement using generalizations of histogram equalization", IEEE Transactions on Image Processing, Vol. 9, No. 5, pp. 889-896, 2000.

[3] J.Y. Kim, L.S. Kim, and S.H. Hwang, "An advanced contrast enhancement using partially overlapped sub-block histogram equalization", IEEE Transactions on Circuits Systems for Video Technology, Vol. 11, No. 4, pp. 475-484, 2001.

[4] Y.Y. Schechner, S.G. Narasimhan, and S.K. Nayar, "Instant dehazing of images using polarization", In: Proc. of the IEEE Conference on Computer Vision and Pattern Recognition, Vol. 1, pp 325-332, 2001.

[5] S.Shwartz, E.Namer, and Y.Y. Schechner, "Blind haze separation", In: Proc. of the IEEE Conference on Computer Vision and Pattern Recognition, Vol. 2, pp. 1984-199, 2006.

[6] T. Treibitz and Y.Y. Schechner, "Polarization: Beneficial for visibility enhancement?", In: Proc. of the IEEE Conference on Computer Vision and Pattern Recognition, pp. 525-532, 2009.

[7] S.G. Narasimhan and S.K. Nayar, "Chromatic framework for vision in bad weather", In: Proc. of the IEEE Conference on Computer Vision and Pattern Recognition, Vol. 1, pp. 598-605, 2000.

[8] S.G. Narasimhan and S.K. Nayar, "Contrast restoration of weather degraded images", IEEE
Transactions on Pattern Analysis and Machine Intelligence, Vol. 25, No.6, pp.713-24, 2003.

[9] C.L. Hung, Z. Ma, C.Y. Lin, H.H. Wang, "Image Haze Removal of Optimized Contrast Enhancement Based on GPU", Frontier Computing, pp. 53-63, 2016.

[10] J. Kopf, B. Neubert, B. Chen, M. Cohen, D. Cohen-Or, O. Deussen, M. Uyttendaele, and D. Lischinski, "Deep photo: Model-based photograph enhancement and viewing," $A C M$ Transactions on Graphics, Vol. 27, No. 5, p. 116, 2008.

[11] E. Alharbi, P. Ge, and H. Wang, "A Research on Single Image Dehazing Algorithms Based on Dark Channel Prior", Journal of Computer and Communications, Vol. 4, pp.47-55, 2016.

[12] R. Tan, "Visibility in bad weather from a single image", In: Proc. of the IEEE Conference on Computer Vision and Pattern Recognition, pp. $1-8,2008$.

[13] P. Chavez, "An improved dark-object substraction technique for atmospheric scattering correction of multispectral data", Remote Sensing of Environment, Vol. 24, No. 3, pp .459-479, 1988.

[14] R. Fattal, "Single image dehazing", $A C M$ Transactions on Graphics, Vol. 27, No. 72, pp.1-9, 2008.

[15] K. He, J. Sun, and X. Tang, "Single image haze removal using dark channel prior," IEEE Trans. Pattern Analysis and Machine Intelligence, Vol. 33, No. 12, pp. 2341-2353, 2011.

[16] M.I. Anwar and A. Khosla, "Vision enhancement through single image fog removal", International Journal of Engineering Science and Technology, Vol. 20, No. 3, pp. 1075-1083, 2017.

[17] K. Nishino, L. Kratz, and S. Lombardi, "Bayesian defogging", International Journal of Computer Vision, Vol. 98, No. 3, pp.263-278, 2012.

[18] G.F. Meng, Y. Wang, J.Y. Duan, S.M. Xiang and C.J. Pan, "Efficient image dehazing with boundary constraint and contextual regularization", In: Proc. of the IEEE International Conference on Computer Vision, pp. 617-624, 2013.

[19] K. Tang, J. Yang, and J. Wang, "Investigating haze-relevant features in a learning framework for image dehazing", In: Proc. of the IEEE Conference on Computer Vision and Pattern Recognition, pp. 2995-3000, 2014.

[20] Q. Zhu, J. Mai, and L. Shao, "A fast single image haze removal algorithm using color 
attenuation prior", IEEE Transactions on Image Processing, Vol. 24, No. 11, pp. 3522-33, 2015.

[21] E. J. McCartney, Optics of the atmosphere: scattering by molecules and particles, New York, John Wiley and Sons, Inc. 1976.

[22] H. Koschmieder, Theorie der horizontalen sichtweite, Beitr. Phys. Freien Atm., Vol. 12 pp.171-181, 1924.

[23] www.nmt.edu/tcc'

http://infohost.nmt.edu/tcc/help/pubs/colortheor y/web/hsv.html

[24] J.P. Tarel, N. Hautiere, A. Cord, D. Gruyer, and H. Halmaoui, "Improved Visibility of Road Scene Images under Heterogeneous Fog", In: Proc. of IEEE Intelligent Vehicles Symposium, pp. 478-485, 2010.

[25] J.P. Tarel, N. Hautière, L. Caraffa, A. Cord, H. Halmaoui, and D. Gruyer, "Vision Enhancement in Homogeneous and Heterogeneous Fog", IEEE Intelligent Transportation Systems Magazine, Vol. 4 , No. 2, pp. 6-20, 2012. 